\title{
TENOTOMIA DO MÚSCULO FLEXOR DIGITAL SUPERFICIAL E DESMOTOMIA ACESSÓRIA EM EQUINOS: GONIOMETRIA RADIOMETACARPIANA, METACARPOFALÂNGICA E INTERFALÂNGICA
}

\author{
Carlos Alberto Hussni, ${ }^{1}$ Marcos Jun Watanabe, ${ }^{2}$ Ana Liz Garcia Alves, ${ }^{3}$ \\ José Luiz de Mello Nicoletti ${ }^{4}$ e Luiz Carlos Vulcano ${ }^{5}$
}

\author{
1. Professor adjunto da FMVZ/Unesp, Botucatu, SP. E-mail: cahussni@fmvz.unesp.br \\ 2. Professor substituto, DsC., FMV/Unesp \\ 3. Professora assistente, doutora da FMV/Unesp \\ 4. Professor adjunto da FMV/Unesp \\ 5. Professor livre-docente da FMV/Unesp.
}

\section{RESUMO}

A proposta deste estudo foi avaliar os efeitos da tenotomia do músculo flexor digital superficial e a desmotomia do seu ligamento acessório sobre os ângulos formados pelas faces dorsais radiometacarpiano, metacarpofalângico e interfalângicos proximal e distal em equinos. Realizaram-se em nove equinos a tenotomia do músculo flexor digital superficial no membro torácico direito (MTD) e a desmotomia de seu ligamento acessório no membro torácico esquerdo (MTE). A goniometria radiológica foi procedida no pré-operatório e aos quinze, trinta e sessenta dias após as cirurgias. A tenotomia praticada no MTD diminuiu significativa- mente o ângulo metacarpofalângico (média com desvio-padrão pré-operatório $-140,7 \pm 6,85$; sessenta dias $\left.-128,2^{\circ} \pm 5,93\right)$ e aumentou o interfalângico distal (média com desvio-padrão préoperatório $-180,2^{\circ} \pm 5,43$; sessenta dias $\left.-197,3^{\circ} \pm 8,29\right)$. A desmotomia realizada no MTE diminuiu significativamente o ângulo interfalângico proximal (média com desvio-padrão pré-operatório $-172,6 \pm 1,87$; sessenta dias $-167,6 \pm 1,36$ ). As cirurgias estudadas não interferiram nos eixos radiometacarpianos. Os procedimentos cirúrgicos estudados alteraram significativamente os ângulos articulares distais ao carpo.

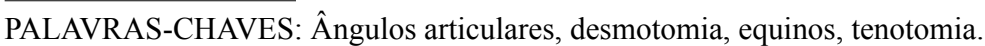

\section{ABSTRACT}

\section{SUPERFICIAL DIGITAL FLEXOR TENOTOMY AND SUPERIOR CHECK LIGAMENT DESMOTOMY IN HORSES: RADIO- METARCARPAL, METACARPOPHALANGEAL AND INTERPHALANGEAL GONIOMETRY}

The purpose of this study was to investigate the effects of the superficial digital tenotomy and the superior check ligament desmotomy on the radio-metacarpal, metacarpophalangeal, proximal and distal interphalangeal angles in horses. Under general anesthesia the superficial digital flexor tenotomy and superior check ligament desmotomy were performed, respectively, on the right and left forelimbs in nine horses. Before surgery and on $15^{\text {th }}, 30^{\text {th }}$, and $60^{\text {th }}$ postoperative days the radio-metacarpal, metacarpophalangeal, and proximal and distal interphalangeal angles were measured by radiographic examination. Tenotomy decreased significantly the metacarpophalangeal angle (mean \pm standard deviation: preoperative $-140.7^{\circ} \pm 6.85 ; 60^{\text {th }}$ day $-128.2^{\circ}$ \pm 5.93 ) and increased the distal interphalangeal angle (mean \pm standard deviation: preoperative $-172.6 \pm 1.87 ; 60^{\text {th }}$ day -167.6 \pm 1.36 ). Desmotomy decreased the proximal interphalangeal angle (mean \pm standard deviation: preoperative $-180.2^{\circ} \pm 5.43$; 60th 
day- $\left.197.3^{\circ} \pm 8.29\right)$. These surgical procedures did not change the radio-metacarpal angle. The superficial digital flexor tenotomy

KEY WORDS: Desmotomy, horses, joint angles, tenotomy.

\section{INTRODUÇÃO}

Nos equinos, o músculo flexor digital superficial tem como função a flexão digital e carpal (GETTY, 1981; BUDRAS e RÖCK, 1994; WISSDORF et al., 1998). Pela localização, ao executar a flexão digital esse tendão atua de modo evidente e direto na flexão da articulação metacarpofalângica, tracionando a articulação interfalângica proximal, resultando ainda na flexão do carpo. Esses movimentos são estabilizados pelas estruturas relacionadas como o ligamento anular, o canal carpal e em destaque maior o próprio ligamento acessório (frenador carpiano superior), permitindo equilíbrio de forças e evitando a hiperextensão (SHIVELY, 1983; WISSDORF et al., 1998).

Diversos são os relatos, as descrições e as recomendações sobre a deformidade flexora nos equinos como sendo enfermidade envolvendo os tendões dos músculos flexores digitais, com a impossibilidade de se realizar a extensão e angular-se normalmente a articulação metacarpofalângica ou as interfalângicas. De acordo com a intensidade ou grau de deformidade apresentada, essa enfermidade tem por característica primordial a manutenção do ângulo da face dorsal da articulação metacarpofalângica acima da angulação normal (SEIDEL, 1979; HERMANS, 1992).

$\mathrm{Na}$ deformidade flexora comprometendo a articulação metacarpofalângica são indicadas a tenotomia do músculo flexor digital superficial bem como a desmotomia de seu ligamento acessório como técnicas para o tratamento cirúrgico (FRANK, 1964; NÈMETH, 1976; MCLAUGHLIN, 1978; HICKMAN, 1979; BLACKWELL, 1983; FACKELMAN, 1983; DIETZ, 1985; WAGNER et al., 1985; DULAC, 1987; STASHAK, 1987; GERRING, 1989; WAGNER, 1990; BARR, 1994) e ainda empregado nas tendinites (COTE et al., 1994; FULTON et al., 1994; HOGAN \& BRAMLAGE, 1995). A tenotomia permite maior liberação do músculo, podendo entretanto resultar em aspectos funcionais e estéticos por vezes indesejáveis (BLACKWELL, 1983; FACKELMAN, 1983; STASHAK, 1987; WAGNER, 1990; BARR, 1994). and the superior check ligament desmotomy cause different and significant changes in joint angles distal to the carpus.

Considerando-se a origem proximal do músculo flexor digital superficial e a inserção deste na porção das falanges proximal e média, o resultado da tenotomia do flexor digital superficial e a desmotomia de seu ligamento acessório poderiam interferir significativamente no membro operado. Isto analisado, objetivou-se estudar as variações angulares do eixo radiometacarpiano e das articulações metacarpofalângica, interfalângicas proximal e distal, aspectos estes analisados e comparados na tenotomia do músculo flexor digital superficial e na desmotomia do acessório deste tendão.

\section{MATERIAL E MÉTODOS}

Os procedimentos utilizados na experimentação estão de acordo com as normas nacionais que estabelecem a ética na experimentação animal, aprovado pela Câmara de Ética em Experimentação Animal da FMVZ - UNESP - Botucatu, no 052/2002.

Em nove equinos adultos hígidos, sem raça definida, de ambos os sexos, com peso médio de $380 \mathrm{~kg}$, foram realizadas a tenotomia do músculo flexor digital superficial no membro torácico direito (MTD) e a desmotomia do acessório do superficial (superior check ligament) no membro torácico esquerdo (MTE).

Uma semana antes do início do experimento, procedeu-se ao casqueamento dos animais. No dia anterior às cirurgias, foram realizados exames radiográficos de ambos os membros torácicos de cada animal, com aparelho portátil ${ }^{1}(60 \mathrm{kV}-5 \mathrm{mAs})$, com a distância foco-filme de $70 \mathrm{~cm}$, procedendo-se com filmes ${ }^{2} 30 \mathrm{X} 40$ $\mathrm{cm}$ montados em chassi com ecran verde, ${ }^{3}$ revelados em equipamento automático. ${ }^{4}$

O membro torácico a ser radiografado foi apoiado sobre prancha de madeira de modo a distanciar a sola do piso, mantendo-se sua posição ortostática. $\mathrm{O}$ membro contralateral foi flexionado, removendo-o do

\footnotetext{
1. FNX - 90CTI - Electra Ltda.

2. Filme Radiográfico - Kodak Brasileira Comúsculo Ind. Ltda.

3. Ecran - Kodak Brasileira Comúsculo Ind. Ltda.

4. MX2 - Macrotec Ltda.
} 
apoio. Dessa forma, todo o apoio dos membros torácicos recaiu sobre o membro a ser radiografado, o que conferiu, por princípio, máxima carga sobre o membro em apoio com o animal em estação. Com o membro torácico a ser radiografado apoiado, procederam-se às radiografias buscando-se o eixo radiometacarpiano, com o chassi posicionado na face medial do carpo, abrangendo, este, parte da diáfise radial e parte da diáfise metacarpiana principal. Com o chassi posicionado na face medial da porção distal do membro, abrangeram-se parte da diáfise metecarpiana e as três falanges, incluindo o casco (Figura 1).

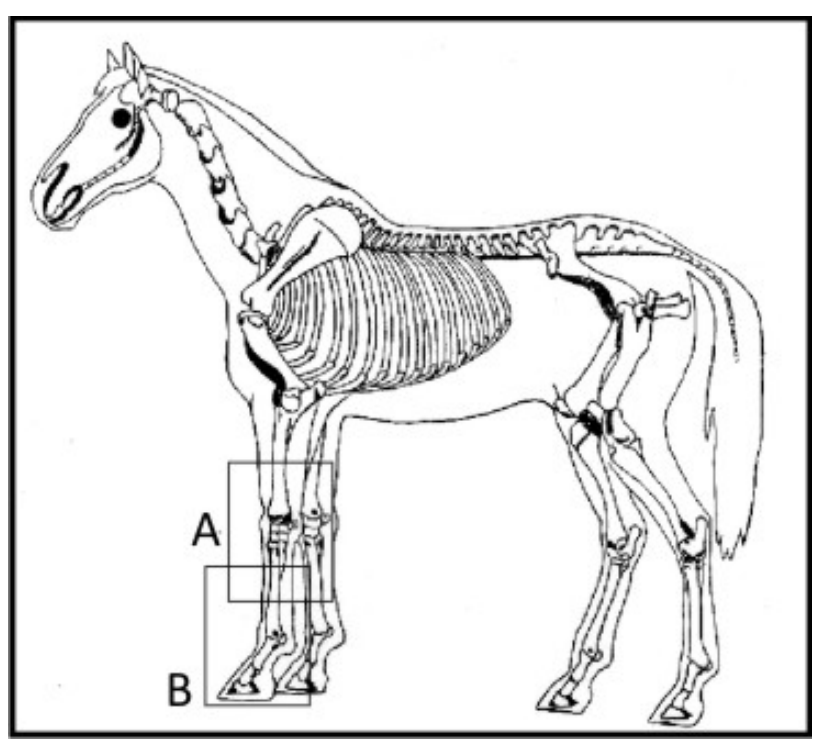

FIGURA 1. Desenho demonstrativo das estruturas radiografadas em exposição lateromedial.

A - chassi posicionado centrado na face medial do carpo, abrangendo a diáfise distal do rádio e a diáfise proximal do metacarpo;

B - chassi posicionado abrangendo a diáfise metecarpiana distal e as três falanges.

Ambas as radiografias foram realizadas no sentido lateromedial, posicionando-se o aparelho de raio $\mathrm{X}$ a $50 \mathrm{~cm}$ entre a ampola e o chassi.

Após jejum alimentar e hídrico de 24 horas, cada animal recebeu medicação pré-anestésica com 0,05 $\mathrm{mg} / \mathrm{kg}$ de acepromazina ${ }^{5}$ via intravenosa. Seguiu-se a tricotomia na face medial da região distal do rádio esquerdo e no membro torácico direito nas faces palmar e lateral do metacarpo.

5. Acepran 1\% - Univet S.A.
O animal a ser operado teve a anestesia geral inalatória induzida com éter gliceril guaiacol ${ }^{6}(100 \mathrm{mg} /$ $\mathrm{kg})$ e tiopental sódico ${ }^{7}(5 \mathrm{mg} / \mathrm{kg})$ via intravenosa, e mantida com halotano ${ }^{8}$, sendo posicionado em decúbito dorsal.

Após preparação de rotina, a tenotomia do músculo flexor digital superficial foi efetuada com acesso pela face lateral do terço médio do metacarpo, com incisão da pele de aproximadamente $3 \mathrm{~cm}$ entre os tendões dos músculos flexores digitais. Aberta a fáscia subcutânea, esta foi ampliada com auxílio de tesoura. Isolou-se o tendão músculo flexor digital superficial com pinça hemostática curva tipo Kelly e procedeu-se à secção transversal do tendão com bisturi. A seguir, a fáscia foi suturada com fio de poliglactina ${ }^{9} \mathrm{em}$ pontos $\mathrm{X}$, e a pele com náilon ${ }^{10}$ com pontos tipo Wolff.

A desmotomia do acessório do superficial, realizada em ato contínuo, foi semelhante à técnica já descrita anteriormente (AUER \& STICK, 1999), tendo por acesso a face medial da porção distal do rádio, com incisão da pele, mediante a utilização de bisturi, de aproximadamente $10 \mathrm{~cm}$, cranial e paralela à veia cefálica do antebraço. Seguiu a abertura da fáscia do músculo flexor carpo radial. A secção do ligamento acessório foi iniciada com bisturi e completada com tesoura. Suturaram-se a fáscia com fio de poliglactina em padrão cerzidura e a pele com náilon, com pontos tipo Wolff.

Trataram-se as feridas cirúrgicas a cada 48 horas com tintura de iodo, ${ }^{11}$ seguida da aplicação de penso protetor com bandagens, até o $12^{\circ}$ dia pós-operatório, quando foram retirados os pontos de pele. No dia das cirurgias e a cada 72 horas, os animais foram medicados com $20.000 \mathrm{UI} / \mathrm{kg}$ de penicilina benzatina associada. ${ }^{12}$ Cada animal recebeu ainda no dia das cirurgias e a cada doze horas, durante cinco dias, fenilbutazona ${ }^{13}$ $(2,2 \mathrm{mg} / \mathrm{kg})$, via intravenosa.

Exames radiográficos foram executados, semelhantes aos realizados nos momentos pré-operatório, aos quinze, trinta e sessenta dias após as cirurgias.

6. Eter Gliceril Guaiacol - Henrifarma Ltda.

7. Tiopental - Cristália Ltda.

8. Halotano -Cristália Ltda.

9. Vicril 2-0 - Johnson \& Johnson Ltda.

10. Mononylon 1 - Brasmédica S.A.

11. Tintura de Iodo $2 \%$ - Rioquimica Ltda.

12. Pentabiótico Veterinário - Fort Dodge Ltda.

13. Butazolidina - Novartis S.A. 
O contorno dos ossos foi obtido em cada radiografia, apoiando-se sobre esta papel translúcido para possibilitar o traçado a lápis do contorno das imagens, de acordo com cada posição.

No papel contendo o desenho do contorno dos ossos observados em cada radiografia, efetuaram-se segmentos de retas no eixo diafisário referente a cada osso (Figura 2). Cada goniometria foi realizada a partir da face dorsal das junções ósseas, estas medidas em graus utilizando-se transferidor, determinando-se, assim, o ângulo formado pelo eixo radiometacarpiano (ARM) e os ângulos articulares metacarpofalângico (AMF), interfalângico proximal (AIP) e interfalângico distal (AID) de ambos os membros, de todos os animais nos quatro momentos.

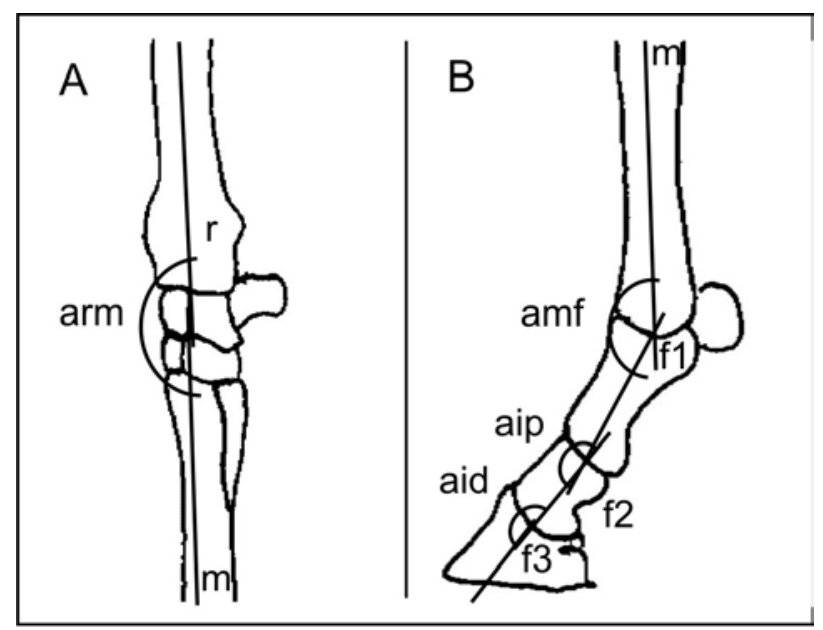

FIGURA 2. Diagrama demonstrativo da obtenção do ângulo formado pelo eixo radiometacarpiano (A) e dos ângulos formados pelas articulações metacarpofalângica e interfalângicas (B), obtido a partir de exames radiográficos $(\mathbf{r}$ - rádio, $\mathbf{m}$ - metacarpo, $\mathbf{f 1}$ - falange proximal, $\mathbf{f} \mathbf{2}$ - falange média, $\mathbf{f} 3$ - falange distal, arm - ângulo do eixo radiometacarpiano, amf - ângulo da articulação metacarpofalângica, aip - ângulo da articulação interfalângica proximal, aid - ângulo da articulação interfalângica distal).

Aplicou-se a análise estatística para as medidas dos ângulos dos eixos e articulações nos vários momentos de avaliação do experimento. Os membros direito e esquerdo foram comparados em cada momento utilizando-se a análise multivariada de perfil (MORRISON, 1990), que possibilitou ainda a comparação dos ângulos entre momentos para cada membro e respectivo procedimento cirúrgico (direito - tenoto- mia; esquerdo - desmotomia). O nível de significância adotado foi de $5 \%$.

\section{RESULTADOS}

As posições radiográficas utilizadas no sentido lateromedial, em cada membro torácico de cada animal nos quatro momentos, permitiram os traçados dos contornos ósseos, destes a determinação dos eixos diafisários e a mensuração em graus do eixo radiometacarpiano e das articulações metacarpofalângica e interfalângica de cada membro.

Sobre os ângulos mensurados (Tabela 1), as médias dos eixos radiometacarpianos (ARM) diferiram entre os membros direito (tenotomia) e esquerdo (desmotomia), sendo maior o ângulo formado pelo membro esquerdo mensurado aos trinta dias (ARM direito $=168,8^{\circ}$ e $A R M$ esquerdo $=171,2^{\circ}$ ) e aos sessenta dias $\left(\right.$ ARM direito $=169,8^{\circ}$ e ARM esquerdo $=171,9^{\circ}$ ) após as cirurgias.

O ângulo formado pela articulação metacarpofalângica do membro direito diminuiu do préoperatório $\left(140,7^{\circ}\right)$ para os momentos pós-operatórios (quinze dias $=126,9^{\circ}$; trinta dias $=126,6^{\circ}$; sessenta dias $=128,2^{\circ}$ ). Não houve diferenças entre os momentos desta articulação no membro esquerdo. Entre os membros direito e esquerdo, observaram-se diferenças com maior ângulo formado no membro esquerdo aos quinze, trinta e sessenta dias após as cirurgias (quinze dias - direito $=126,9^{\circ}$, esquerdo $=138,0^{\circ}$; trinta dias - direito $=126,6^{\circ}$, esquerdo $=137,5^{\circ}$; sessenta dias direito $=128,2^{\circ}$, esquerdo $=140,0^{\circ}$ )

As articulações interfalângicas proximais do membro direito submetido à tenotomia do músculo flexor digital superficial não variaram significativamente entre os momentos, diferindo do observado na articulação em questão no membro esquerdo, este submetido à desmotomia do acessório do superficial, em que ocorreu redução entre os momentos pré-operatório e sessenta dias (AIP membro esquerdo - pré $=172,6^{\circ}$; sessenta dias $\left.=167,6^{\circ}\right)$. Não ocorreram diferenças entre os membros para cada momento.

Para o membro direito submetido à tenotomia do flexor digital superficial, o ângulo interfalângico distal elevou-se do momento pré-operatório $\left(180,2^{\circ}\right)$ para os momentos quinze dias $\left(199,3^{\circ}\right)$, trinta dias $\left(196,4^{\circ}\right)$ e sessenta dias $\left(197,3^{\circ}\right)$ após a cirurgia. Para 
o membro esquerdo, os ângulos dessas articulações não variaram entre os momentos. $\mathrm{O}$ ângulo da articulação interfalângica distal mostrou-se diferente entre os membros direito e esquerdo, com valores superiores para o membro direito nos momentos quinze dias (direito $=199,3^{\circ}$, esquerdo $\left.=183,8^{\circ}\right)$, trinta dias (direito $=196,4^{\circ}$, esquerdo $=186,3^{\circ}$ ) e sessenta dias $\left(\right.$ direito $=197,3^{\circ}$, esquerdo $=182,2^{\circ}$ ) após a cirurgia.

TABELA 1. Médias e desvio-padrão de nove repetições do ângulo (graus) do eixo radiometacarpiano (ARM), articulações metacarpofalângica (AMF), interfalângica proximal (AIP) e interfalângica distal (AID) segundo membros direito (tenotomia do flexor digital superficial) e esquerdo (desmotomia do acessório) nos quatro momentos.

\begin{tabular}{lcccc}
\hline \multicolumn{3}{c}{ Momentos } \\
\hline Variável & Pré-operatório & Quinze dias & Trinta dias & Sessenta dias \\
\hline ARM direito & $172,5 \pm 2,26 \mathrm{Aa}$ & $170,9 \pm 5,55 \mathrm{Aa}$ & $168,8 \pm 2,61 \mathrm{Aa}$ & $169,8 \pm 1,80 \mathrm{Aa}$ \\
ARM esquerdo & $172,1 \pm 2,07 \mathrm{Aa}$ & $171,8 \pm 2,88 \mathrm{Aa}$ & $171,2 \pm 1,95 \mathrm{Ab}$ & $171,9 \pm 1,82 \mathrm{Ab}$ \\
AMF direito & $140,7 \pm 6,85 \mathrm{Aa}$ & $126,9 \pm 5,45 \mathrm{Ba}$ & $126,6 \pm 6,58 \mathrm{Ba}$ & $128,2 \pm 5,93 \mathrm{Ba}$ \\
AMF esquerdo & $140,2 \pm 3,67 \mathrm{Aa}$ & $138,0 \pm 5,72 \mathrm{Ab}$ & $137,5 \pm 6,36 \mathrm{Ab}$ & $140,0 \pm 6,40 \mathrm{Ab}$ \\
AIP direito & $171,4 \pm 2,76 \mathrm{Aa}$ & $168,2 \pm 4,92 \mathrm{Aa}$ & $169,4 \pm 3,74 \mathrm{Aa}$ & $169,1 \pm 3,35 \mathrm{Aa}$ \\
AIP esquerdo & $172,6 \pm 1,87 \mathrm{Aa}$ & $170,3 \pm 3,13 \mathrm{ABa}$ & $169,6 \pm 3,79 \mathrm{ABa}$ & $167,6 \pm 1,36 \mathrm{Ba}$ \\
AID direito & $180,2 \pm 5,43 \mathrm{Aa}$ & $199,3 \pm 5,96 \mathrm{Ba}$ & $196,4 \pm 7,27 \mathrm{Ba}$ & $197,3 \pm 8,29 \mathrm{Ba}$ \\
AID esquerdo & $182,9 \pm 5,90 \mathrm{Aa}$ & $183,8 \pm 8,90 \mathrm{Ab}$ & $186,3 \pm 3,22 \mathrm{Ab}$ & $182,2 \pm 4,74 \mathrm{Ab}$ \\
\hline
\end{tabular}

Para cada membro, médias de momentos seguidas de pelo menos uma letra igual não diferem significativamente $(\mathrm{P}>0,05)$. Letras maiúsculas comparam os momentos e minúsculas comparam os membros.

\section{DISCUSSÃO}

A tenotomia do flexor digital superficial e a desmotomia do acessório do superficial são técnicas operatórias bastante estudadas, recomendadas e aplicadas no restabelecimento de animais portadores de enfermidades que acometem os membros dos equinos, destacando-se as deformidades flexoras envolvendo a articulação metacarpofalângica (FRANK, 1964; NÈMETH, 1976; McLAUGHLIN, 1978; HICKMAN, 1979; BLACKWELL, 1983; FACKELMAN, 1983; DIETZ, 1985; WAGNER et al., 1985; DULAC, 1987; STASHAK, 1987; GERRING, 1989; WAGNER, 1990; BARR, 1994; DYSON, 1997; McILWRAITH, 2002). Essas técnicas são ainda empregadas nas tendinites (COTE et al., 1994; FULTON et al., 1994; HENNINGER, 1994; HOGAN \& BRAMLAGE, 1995), melhorando o prognóstico do desempenho atlético nesses equinos. Trata-se de técnicas que foram exequíveis com o animal posicionado em decúbito dorsal conforme realizado neste experimento, considerando-se que a literatura citada recomenda o decúbito lateral para tais procedimentos.
A obtenção dos ângulos do eixo radiometacarpiano e das articulações metacarpofalângica e interfalângicas de cada membro operado consistiu em método simples, de fácil execução e de satisfatória acurácia, concordante com a descrição de métodos semelhantes (EMERY et al., 1977; BUSHE et al., 1988; HUSSNI et al., 1996). Outros métodos goniométricos foram também eficientes dentro da proposta a que serviram, tendo como destaque o uso de goniômetros (SHOEMAKER et al., 1991; SAVELBERG et al., 1997; ALEXANDER et al., 2001; LAGE et al., 2009), que, em hipótese ora não estudada, podem ter menor precisão ao se avaliar variações angulares quando comparados com o método obtido a partir de exames radiográficos. Porém, a metodologia de mensuração angular com radiografias pode compreender somente das articulações distais até as articulações carpais e tarsais, sendo este método inviável nas articulações mais altas como a escapuloumeral e coxofemoral.

Os valores médios obtidos do eixo radiometacarpiano (ângulo do carpo) que apresentaram a maior variação entre $172,5^{\circ}$ no pré-operatório e $168,8^{\circ}$ aos trinta dias para o membro direito, este considerado 
ainda como ângulo do carpo, ficaram próximos ao descrito com o animal e apoio quadrupedal (SEIDEL, 1979) e semelhante ao estudado em peças anatômicas submetidas à ação de forças (SHOEMAKER et al., 1991). Considere-se que, para este parâmetro e para os demais ângulos mensurados, o exame radiográfico foi realizado com o apoio do animal com sobrecarga máxima de peso sobre o membro, estando apoiado com a parte torácica do animal somente no membro submetido, podendo, portanto, diferir de outra mensuração com o animal em apoio quadrupedal.

O ângulo articular metacarpofalângico do membro direito teve redução significativa, o que não ocorreu com o membro esquerdo, demonstrando que a tenotomia do músculo flexor digital superficial atua intensamente na redução deste ângulo articular. Apesar de a desmotomia do acessório do superficial corrigir a deformidade flexora, neste estudo, essa técnica não reduziu significativamente o ângulo metacarpofalângico. Isso deve-se, principalmente, à função moderadora desse ligamento acessório e da função ativa e de maior efetividade dos tendões flexores digitais e do ligamento suspensório do boleto, estes ativos e preservados, com a observação evidente da interação estrutural entre as articulações e eixos estudados e a interação destes como sistema integrado envolvendo inclusive o estojo córneo (EMERY, 1977; WISSDORF et al., 1998; DENOIX et al., 1999; ALEXANDER et al., 2001).

O valor médio em graus obtido das articulações metacarpofalângicas de ambos os membros no momento pré-operatório, considerado normal, foi discretamente abaixo do divulgado pela maioria dos autores (144 a 150 graus) (SEIDEL, 1979; SHOEMAKER et al., 1991; LAGE et al., 2009). Deve-se isso à metodologia do procedimento radiológico, considerando que, neste momento, o membro radiografado apoiava o solo e o membro contralateral estava suspenso e sem apoio, o que gerou maior carga de peso sobre o membro radiografado e assim diminuição do ângulo metacarpofalângico, porém de modo discreto. De modo semelhante ocorreu a diminuição deste ângulo em estudo com cargas crescentes de força realizado em peças anatômicas (SHOEMAKER et al., 1991).

Ao se observar os ângulos das articulações interfalângicas proximal e distal em ambos os membros dos animais operados, evidenciam-se alterações destes ângulos de acordo com a cirurgia realizada.
Fica evidente a redução do ângulo interfalângico proximal decorrente da desmotomia do acessório do superficial, possivelmente em decorrência da eliminação da ação do ligamento acessório causando maior tração pelo tendão flexor digital superficial em sua inserção na porção distal palmar da falange proximal e da porção proximal palmar da falange média. Estudos das resultantes de forças que atuam na articulação em questão explicariam mais claramente essa redução angular.

Sobre a articulação interfalângica distal, as medidas dos ângulos elevaram-se quando comparados os momentos pré-operatório e os demais momentos para o membro direito, sem diferenças para o membro esquerdo. Com base no descrito e observando-se a anatomia funcional da região (WISSDORF et al., 1998; DENOIX et al., 1999), deve ser considerada a sobrecarga sofrida pelo tendão do músculo flexor digital profundo na força exercida pela articulação metacarpofalângica, dada a ausência da ação do músculo flexor digital superficial após a tenotomia. Ao aumentar-se a tensão sobre o tendão do músculo flexor digital profundo ocorre flexão da articulação interfalângica distal, aumentando, portanto, o ângulo desta. Isto pôde ser confirmado por analogia inversa, ao observar-se a diminuição do ângulo articular metacarpofalângico e a elevação dos ângulos articulares interfalângicos proximal e distal ao elevar-se o talão dos cascos (BUSHE et al., 1988).

Aplicando-se metodologias semelhantes na goniometria articular com base em imagens radiográficas, a realização das radiografias em apoio monopedal anterior decorre em maior carga de peso sobre o membro, podendo ocorrer diferenças se comparar-se à realização dos exames radiográficos com o animal e apoio quadrupedal (HUSSNI et al., 1996), sendo isto hipótese e não objeto de estudo.

\section{CONCLUSÕES}

A tenotomia do flexor digital superficial em equinos diminui o ângulo metacarpofalângico e eleva o ângulo interfalângico distal. A desmotomia do acessório do flexor digital superficial diminui o ângulo interfalângico proximal. A tenotomia do flexor digital superficial e a desmotomia do acessório deste tendão não alteraram o eixo radiometacarpiano. 


\section{AGRADECIMENTO}

Ao Prof. Dr. Adalberto José Crocci (in memoriam).

\section{REFERÊNCIAS}

ALEXANDER, G. R.; GIBSON, K. T.; DAY, R. E.; ROBERTSON, I. D. Effects of superior check desmotomy on flexor tendon and suspensory ligament strain in equine cadaver limb. Veterinary Surgery, v. 30, p. 522-527, 2001.

AUER, J. A.; STICK, J. A. Equine surgery. 2. ed. Philadelphia: W. B. Saunders, 1999. 937 p.

BARR, A. Developmental flexural deformities in the horse. In Practice, v. 16, p. 182-188, 1994.

BLACKWELL, R. B. Response of acquired flexure deformity of the metacarpophalangeal joint to desmotomy of the inferior check ligament. In: ANNUAL AAEP CONVENTION, 26., 1982, Georgia. Proceedings... Athens, 1983. p. 107-111.

BUDRAS, K-D.; RÖCK, S. Atlas der Anatomie des Pferdes: Lehrbuch für Tierärzte und Studierende. 2. ed. Hannover: Schlütersche, 1994. 144 p.

BUSHE, T.; TURNER, T. A.; POULOS, P. W.; HARWELL, N. M. The effect of hoof angle on coffin, pastern and fetlock joint angles. In: ANNUAL AAEP CONVENTION, 33., 1987, New Orleans. Proceedings... New Orleans:Louisiana, 1988. p. 729-738.

COTE, N.; MARCOUX, M.; LEPAGE, O. M. Complications of desmotomy of the accessory ligament of the superficial digital flexor tendon: a retrospective study of 19 cases (1986-1989). Pratique Vétérinaire Équine, v. 26, p. 103-107, 1994.

DENOIX, J-M. ; BROCHET, J-L. ; HOULIEZ, D. Marechalerie: anatomie, biomécanique, ferrure normal, ferrure spéciale. Paris: Ecole Nationale Vétérinaire d'Alfort, 1999. 210 p.

DIETZ, O. Diagnosis and treatment of congenital contracted tendons in the foal. Monatsheft für Tiermedizin, v. 40, p. 838-340, 1985.

DULAC, O. Affections tendineuses du cheval. Contusions, plaies et sections, luxations, flccidite, retractions, ruptures, désinsertions, tendinites. Pratique Vétérinaire. Équine, v. 19, p. 37-43, 1987.

DYSON, S. J. The musculoskeletal systemúsculo In: ROBINSON, N. E. Current therapy in equine medicine. $4^{\text {th }}$ ed. Philadelphia: Saunders, 1997. p. 1-137.

EMERY, L.; MILLER, J.; VAN HOOSEN, N. Horseshoeing theory and hoof care. Philadelphia: Lea \& Febiger, 1977. 271 p.
FACKELMAN, G. E. Tendon surgery. Veterinary Clinics of North America: Large Animal Practice, v. 5, p. 381-390, 1983.

FRANK, E. R. Veterinary surgery. $7^{\text {th }}$ ed. Minneapolis: Burgess, 1964. $356 \mathrm{p}$.

FULTON, I. C.; MACLEAN, A.; O'RIELLY, J. L.; CHURCH, S. Superior check ligament desmotomy for treatment of superficial digital flexor tendonitis in thoroughbred and standardbred horses. Australian Veterinary Journal, v. 71, p. 233-235, 1994.

GERRING, E. L. Flexural deformities of the limb in foals. Equine Veterinary Education, 1, p. 39-41, 1989.

GETTY, R. Anatomia dos animais domésticos. 5. ed. Rio de Janeiro: Interamericana, 1981. 2000 p.

HENNINGER, R. Treatment of superficial digital flexor tendinitis. Veterinary Clinics of North America: Equine Practice, v. 10, p. 409-424, 1994.

HERMANS, W. A. Hufpflege und Hufbeschlag. Stuttgart: Ulmer, 1992. 269 p.

HICKMAN, J. Contração dos tendões flexores digitais do potro recém-nascido. In: CICLO INTERNACIONALDE CLÍNICA EQÜINA, 2., 1979, São Paulo. Anais... São Paulo, 1979, p. 63-71.

HOGAN, P. M.; BRAMLAGE, L. R. Transection of the accessory ligament of the superficial digital flexor tendon for treatment of tendinitis: long term results in 61 standardbred racehorces (1985-1992). Equine Veterinary Journal, v. 27, p. 221-226, 1995.

HUSSNI, C. A.; NICOLETTI, J. L. M.; THOMASSIAN, A.; GANDOLFI, W.; LUNA, S. P. L.; VULCANO, L. C.; CURI, P. R.; ALVES, A. L. G. Variações dos ângulos das articulações metacarpofalângica e interfalângicas frente a duas técnicas de tenotomia do flexor digital profundo em equinos. Veterinária e Zootecnia, v. 8, p. 27-34, 1996.

LAGE, M. C. G. R.; BERGMANN, J. A. G.; PROCÓPIO, A. M.; PEREIRA, J. C. C.; BIONDINI, J. Associação entre medidas lineares e angulares de equinos a raça Mangalarga Marchador. Arquivo Brasileiro de Medicina Veterinária e Zootecnia, v. 61, p. 968-979, 2009.

McILWRAITH, C. W. Diseases of joints, tendons, ligaments and related structures. In: STASHAK, T. S. Adams' lameness in horses. $5^{\text {th }}$ Philadelphia: Lippincott Williams \& Wilkins, 2002. p. 459-644.

McLAUGHLIN, S. A. Acquired contracted tendons in the foal. Southwest Veterinarian, v. 31, p. 105-106, 1978. 
MORRISON, D. F. Multivariate statistical methods. 3. London: Mc Graw-Hill, 1990. 413 p.

NÈMETH, F. Der tendogenese Stelzfuß beim Fohlen. Der praktischeTierarzt, v. 3, p. 180-181, 1976.

SAVELBERG, H. H. C. M.; BUCHNER, H. H. F.; BECKER, C. K. Recovery of equine forelimb function after desmotomy of the accessory ligament of the deep digital flexor tendon. Equine Veterinary Journal, v. 23, p. 27-29, 1997.

SEIDEL, H. Estudio especial de los movimentos. In: KOLB, E. Fisiologia Veterinária. 2. ed. Zaragoza: Acribia, 1979. p. 914947.

SHIVELY, M. J. Functional and clinical significance of the check ligaments. Equine Practice, v. 5, p. 37-42, 1983.

SHOEMAKER, R. S.; BERTONE, A. L.; MOHAMMAD, L. N.; ARMS, S. W. Desmotomy of the acessory ligament of the super- ficial digital flexor muscle in equine cadaver limb. Veterinary Surgery, v. 20, p. 245-252, 1991.

STASHAK, T. Adam's Lameness in horses. 4. ed. Philadelphia: Lea \& Febiger, 1987. 906 p.

WAGNER, P. C. Flexural deformity of the metacarpophalangeal joint (contracture of the superficial digital flexor tendon). In: WHITE II, N. A.; MOORE, J. N. Current practice of equine surgery. Philadelphia: Lippincott, 1990. p. 476-480.

WAGNER, P. C.; SHIRES, M.; WATROUS, B. J.; KANEPS, A. J.; SCHMOTZER, W. B.; RIEBOLD, T. W. Management of acquired flexural deformity of the metacarpophalangeal joint in Equidae. Journal of the American Veterinary Medical Association, v. 187, p. 915-918, 1985.

WISSDORF, H.; GERHARDS, H.; HUSKAMP, B. Praxisorientierte Anatomie des Pferdes. Hannover: M \& H Schaper Alfeld, $1998.628 \mathrm{p}$.

Protocolado em: 27 fev. 2009. Aceito em: 7 set. 2009. 